\title{
The Effect of Different Surgical Methods on the Number of Circulating Tumor Cells in the Peripheral Blood of Patients with Renal Cell Carcinoma
}

\author{
Dianbin Song1, Zhiyong Wang1* ${ }^{*}$ Xiuming Li ${ }^{1}$, Jingjing Zhang ${ }^{2}$, Qiang Chi ${ }^{1}$, Hui Xu' \\ Hongyang Li ${ }^{1}$, Ying Liu ${ }^{1}$
}

${ }^{1}$ Department of Urology, Affiliated Hospital of Chengde Medical College, Chengde, China

${ }^{2}$ Department of Oncology, Affiliated Hospital of Chengde Medical College, Chengde, China

Email: ^15633142863@163.com

How to cite this paper: Song, D.B., Wang, Z.Y., Li, X.M., Zhang, J.J., Chi, Q., Xu, H., Li, H.Y. and Liu, Y. (2022) The Effect of Different Surgical Methods on the Number of Circulating Tumor Cells in the Peripheral Blood of Patients with Renal Cell Carcinoma. Journal of Cancer Therapy, 13, 1-6. https://doi.org/10.4236/jct.2022.131001

Received: December 15, 2021

Accepted: January 8, 2022

Published: January 11, 2022

Copyright $\odot 2022$ by author(s) and Scientific Research Publishing Inc. This work is licensed under the Creative Commons Attribution International License (CC BY 4.0).

http://creativecommons.org/licenses/by/4.0/

\begin{abstract}
Objective: The objective is to explore the effects of different surgical methodsretroperitoneal laparoscopic radical nephrectomy (RLRN) and open radical nephrectomy (ORN) on the number of circulating tumor cells (CTC) in the peripheral blood of patients with renal cancer. Methods: The clinical data of 63 patients in the Department of Urology, Affiliated Hospital of Chengde Medical College who underwent radical surgery for renal cancer were divided into CTC positive group (18 cases of open surgery and 16 cases of minimally invasive surgery) and CTC negative group (14 cases of open surgery), 15 cases of minimally invasive surgery), overall group ( 32 cases of open surgery, 31 cases of minimally invasive surgery). Observe the changes in the number of CTC 1 week before operation and 1 week after operation. Results: In the positive group, whether it was open surgery or minimally invasive surgery, the postoperative CTC level of patients was significantly reduced $(\mathrm{P}<0.05)$. In the negative group, the CTC changed significantly after minimally invasive surgery $(P<0.01)$, and the CTC level changed indistinctly after open surgery $(\mathrm{P}>0.05)$. In the overall group, both open and minimally invasive surgery CTC decreased significantly, and the difference was statistically significant ( $P$ $<0.05)$. Conclusion: The two different surgical methods can reduce the level of CTC, but compared with ORN, RLRN can significantly increase the number of postoperative CTC. Patients in the CTC-negative group may be less suitable for minimally invasive surgery. CTC levels have certain potential in the selection and guidance of treatment modes for patients with renal cell carcinoma (RCC).
\end{abstract}




\section{Keywords}

Retroperitoneal Laparoscopic Radical Nephrectomy, Open Radical Nephrectomy, Number of Circulating Tumor Cells

\section{Introduction}

Among the malignant tumors of the urinary system, kidney cancer has the highest fatal mortality rate, which accounts for about 40\% [1], and the incidence rate ranks second, second only to bladder cancer [2]. Globally, RCC is increasing with more than 200,000 cases each year, and there are more than 100,000 deaths. Because RCC itself is not sensitive to radiotherapy and chemotherapy, surgery is currently the main treatment method. Early surgical treatment can obtain satisfactory results. With the rapid development of laparoscopic surgery and medical technology, renal cancer is mainly treated with RLRN [3]. But even with early surgical treatment, $20 \%$ - $40 \%$ of patients will have recurrence and distant metastasis after surgery, becoming the main cause of death of kidney cancer [4]. If local recurrence and metastasis can be detected earlier, and surgical resection can be performed in time, the survival rate of RCC patients can be significantly improved [5]. Tumor recurrence and metastasis are achieved by the shedding of tumor cells into the blood circulation, and finally the formation of tumor metastases. With the continuous advancement of molecular biotechnology, especially the clinical application and continuous development of flow cytometry, the cells found in peripheral blood and shed from tumors are first referred to as circulating tumor cells (circulating tumor cells, CTC) [6]. CTC is defined as tumor cells that enter the blood circulation from the tumors primary tumor or metastasis. As a type of tumor cells that are free from the circulatory system, tumor cells can be found earlier than tumor markers and imaging. The level of CTC has been shown to be related to the prognosis of tumors.

\section{Materials and Methods}

\subsection{General Information}

This study retrospectively analyzed 63 RCC patients who visited the Department of Urology at the Affiliated Hospital of Chengde Medical College (hereinafter referred to as our hospital) from June 2018 to December 2019. All patients underwent CTC testing 1 week before and 1 week after surgery. Patients with preoperative peripheral blood CTC counts $\geq 5 / 3.5 \mathrm{~mL}$ are the positive group, and patients with $<5 / 3.5 \mathrm{~mL}$ are the negative group [7].

\subsection{Surgical Method}

1) ORN: Take a waist incision, cut the skin in turn until the kidney is found, ligate and cut off the renal pedicle blood vessel, cut off the ureter, and remove the kidney and tumor. Then suture the cut tissues in sequence. 
2) RLRN: Take the three-hole method at the waist and establish the corresponding channel. Free the renal pedicle vessel, clamp and cut it with hum-lock. Cut the ureter, remove the kidney and tumor, and suture each incision.

3) Index detection methods Collect $3.5 \mathrm{~mL}$ of peripheral venous blood from the patient 1 week before and 1 week after surgery, perform in situ hybridization and fluorescent staining on the cells, and then use a Nikon upright fluorescence microscope to interpret and identify CTC cells, the extraction reagent uses the Human Peripheral Blood Circulating Tumor Cell Negative Enrichment Kit from Scitech Biomedical Technology Company.

\subsection{Statistical Methods}

Use SPSS22.0 statistical software for statistical analysis. The count data are expressed as percentages and rates, and each item is tested for normality and homogeneity of variance. Normally distributed measurement data were compared using the $\chi^{2}$ test, non-normally distributed count data were represented by $\mathrm{Me}$ dian-range, and one-way ANOVA is generally used for differences between groups. Measurement data are expressed as mean \pm standard deviation $(x \pm s)$. $\mathrm{P}$ $<0.05$ indicates that the difference is statistically significant.

\section{Results}

\subsection{There Was No Difference in General Information between the CTC Positive Group and the CTC Negative Group}

Comparing whether the gender, age, and tumor location of the CTC-positive group and CTC-negative group are comparable, it is found that there is no significant difference in general information between the two groups and they are comparable (Table 1).

\subsection{The CTC Level of RCC Can Provide Guidance for the Selection of Surgical Methods}

In order to explore the influence of CTC on the choice of surgical methods for renal cancer patients, we analyzed the changes of CTC before and after different

Table 1. Comparison of general information of patients.

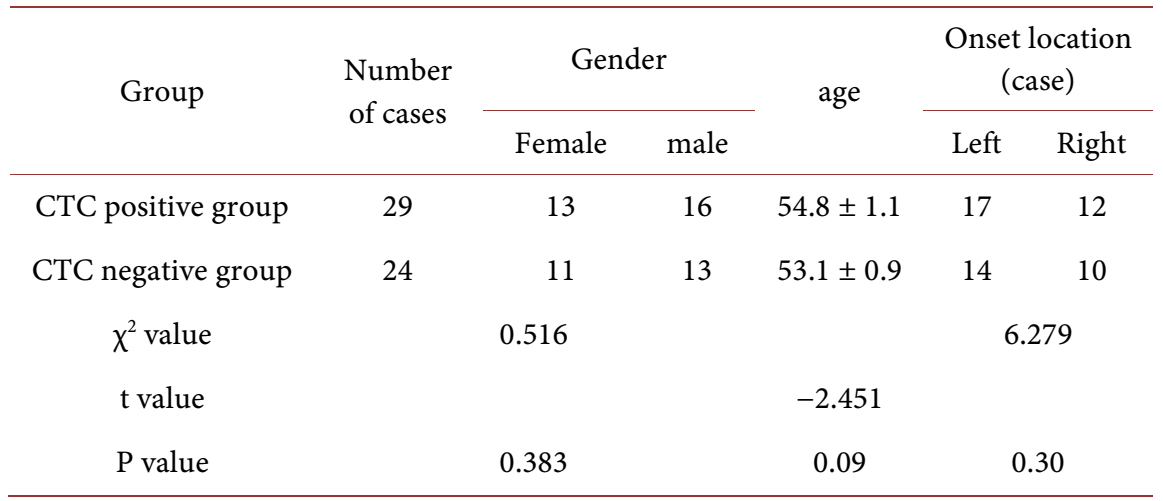

Note: All P values $>0.05$, the difference is not statistically significant. 
surgical methods in the two groups of patients. We found that in the positive group, whether it was open surgery or minimally invasive surgery, the postoperative CTC level was significantly reduced $(\mathrm{P}<0.05)$. In the negative group, the CTC changed significantly after minimally invasive surgery $(\mathrm{P}<0.01)$, and the CTC level changed indistinctly after open surgery $(P>0.05)$. In the overall group, both open and minimally invasive surgery CTC decreased significantly, and the difference was statistically significant $(\mathrm{P}<0.05)($ Table 2$)$.

\section{Discussion}

In recent years, with the rapid development of medical technology and the rise of minimally invasive surgery, compared with ORN surgery, RLRN has begun to be widely used in clinical practice with the advantages of less trauma to patients and faster postoperative recovery and has achieved good therapeutic effects [3]. Compared with ORN, the shortcomings of RLRN compared to ORN are: narrow operating space, gas leaks into the abdominal cavity when the peritoneum is damaged, high technical requirements for operation, and long learning curve. However, RLRN has many advantages, such as clearly showing the anatomical location, less damage, and avoiding the abdominal cavity, organ damage, contamination of the abdominal cavity and opportunities for tumor implantation in the abdominal cavity [8]. In addition, because the posterior abdominal cavity has clear anatomical standards, which significantly improves the efficiency of surgery and reduces the occurrence of surgical complications, the current RLRN mostly chooses the retroperitoneal route [9]. At present, although the RLRN surgical treatment of RCC is quite mature, the surgical benefit of patients with advanced RCC is still limited [10]. The postoperative recurrence rate and metastasis rate are still high, and the treatment effect has not been very good [11]. Therefore, the current early diagnosis of RCC is still an important factor affecting the prognosis.

The detection of CTC can prompt the occurrence of tumors earlier than imaging [12]. Studies have confirmed [13] [14] that the role of CTC in the metastasis

Table 2. Comparison of changes in the number of CTCs before and after operation in each group.

\begin{tabular}{|c|c|c|c|c|c|c|c|c|c|c|c|c|c|c|c|}
\hline & \multicolumn{5}{|c|}{ CTC positive group } & \multicolumn{5}{|c|}{ CTC negative group } & \multicolumn{5}{|c|}{ Overall group } \\
\hline & 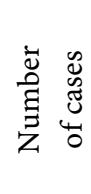 & 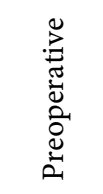 & 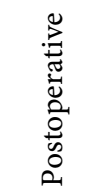 & + & a & 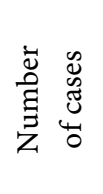 & 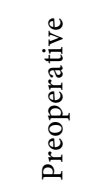 & 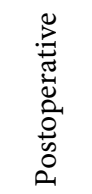 & + & $a$ & 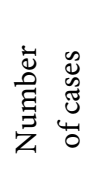 & 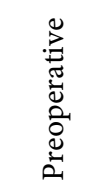 & 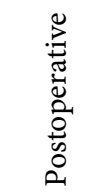 & + & a \\
\hline $\begin{array}{l}\text { ORN } \\
\text { group }\end{array}$ & 18 & $\begin{array}{c}11.301 \\
\pm \\
1.3560\end{array}$ & $\begin{array}{c}6.401 \\
\pm \\
0.8671\end{array}$ & 6.064 & 0.047 & 14 & $\begin{array}{c}3.845 \\
\pm \\
0.2016\end{array}$ & $\begin{array}{c}3.973 \\
\pm \\
1.621\end{array}$ & 0.082 & 0.745 & 32 & $\begin{array}{c}7.201 \\
\pm \\
1.0560\end{array}$ & $\begin{array}{c}3.830 \\
\pm \\
0.7781\end{array}$ & 1.062 & 0.0417 \\
\hline $\begin{array}{l}\text { RLRN } \\
\text { group }\end{array}$ & 16 & $\begin{array}{c}10.314 \\
\pm \\
0.9675\end{array}$ & $\begin{array}{c}4.957 \\
\pm \\
1.2860\end{array}$ & 1.072 & $<0.01$ & 15 & $\begin{array}{c}1.445 \\
\pm \\
0.3116\end{array}$ & $\begin{array}{c}7.273 \\
\pm \\
1.822\end{array}$ & 4.154 & $<0.01$ & 31 & $\begin{array}{c}8.657 \\
\pm \\
1.6860\end{array}$ & $\begin{array}{c}3.173 \\
\pm \\
1.1320\end{array}$ & 2.004 & 0.0210 \\
\hline
\end{tabular}


of breast cancer, prostate cancer, kidney cancer, lung cancer and other diseases has received increasing attention, and its application has been clinically recognized. If the number of CTCs in the peripheral blood of patients with renal cancer can be used to evaluate the progression of the disease, it will have important clinical significance for early intervention in patients with renal cancer. Related studies have found that CTC also plays a role in guiding the choice of tumor treatment [15]. Further study of the interaction between the number of CTCs and the primary tumor may provide a wider field of vision.

Our research found that the CTC level of RCC patients has a certain potential to guide the choice of treatment options, and patients with different CTC levels should choose different surgical methods. Previous studies have confirmed that CTC is positively correlated with poor tumor prognosis [16], and our research has found that either minimally invasive surgery or open surgery can reduce peripheral blood CTC levels, but in the CTC-negative group, minimally invasive surgery CTC counts changed, it's obvious. This indicates that inappropriate surgical methods may affect the prognosis of patients and deserve further attention.

\section{Funded Project}

S\&T Program of Chengde (201904A025).

\section{Conflicts of Interest}

The authors declare no conflicts of interest regarding the publication of this paper.

\section{References}

[1] Attard, G., Swennenhuis, J.F., Oimos, D., et al. (2009) Characterization of ERG, AR and PTEN Gene Status in Circulating Tumor Cells from Patients with Castration-Resistant Prostate Cancer. Cancer Research, 69, 2912-2918.

[2] Deng, K.L., Li, Y.Y., Cui, D.S., et al. (2016) Comparison of Clinical Efficacy between Partial Resection and Radical Resection in the Treatment of pT1bN0M0 Stage Renal Cancer. Cancer Research on Prevention and Treatment, 43, 883-886.

[3] Xu, H. and Yuan, J.L. (2016) Clinical Observation of Retroperitoneal Laparoscopic Radical Nephrectomy for the Treatment of 90 Cases of Renal Cancer. Chinese Journal of Cancer Prevention and Treatment, 23, 176-177.

[4] Yu, J.I., Choi, D.H., Park, W., et al. (2010) Differences in Prognostic Factors and Pattern of Failure between Invasive Micropapillary Carcinoma and Invasive Ductal Carcinoma of the Breast: Matched Case-Control Study. Breast, 19, 231-237.

[5] Zhang, T., Alimu, R., Hou, J.L., et al. (2014) Screening of Circulating Tumor Cells in Peripheral Blood of Renal Cell Carcinoma. Shanxi Medical Journal, 6, 1347-1349.

[6] Wu, J.S., Liu, S.J., Yu, F., et al. (2017) Research Progress in the Detection and Clinical Value of Circulating Tumor Cells in Renal Cell Carcinoma. Journal of Modern Urology, 22, 474-477.

[7] Reid, A.H., Attard, G., Danila, D.C., et al. (2010) Significant and Sustained Antitumor Activity in Post-Docetaxel, Castration-Resistant Prostate Cancer with the CYP17 Inhibitor Abiraterone acetate. Journal of Clinical Oncology, 28, 1489-1495. 
[8] Long, J.C. and Li, X. (2016) The Effect of Retroperitoneal Laparoscopic Radical Nephrectomy on the Body's Cellular Immune Function. Journal of North Sichuan Medical College, 31, 841-843.

[9] Mao, F., Gu, S., Zhong, B., et al. (2017) Effect of Retroperitoneal Laparoscopic Radical Nephrectomy on Patients' Liver and Kidney Function and Myocardial Enzyme Spectrum during Perioperative Period. Journal of Clinical Research, 34, 468-470.

[10] Rasmussen, F. (2013) Metastatic Renal Cell Cancer. Cancer Imaging, 13, 374-380. https://doi.org/10.1102/1470-7330.2013.9035

[11] Na, J.C., Kim, J.H., Kim, S.Y., et al. (2020) Establishment of Patient-Derived Three-Dimensional Organoid Culture in Renal Cell Carcinoma. Investigative and Clinical Urology, 61, 216-223.

[12] Gao, F., Cui, Y., Jiang, H., et al. (2016) Circulating Tumor Cell Is a Common Property of Brain Glioma and Promotes the Monitoring System. Oncotarget, 7, 71330-71340. https://doi.org/10.18632/oncotarget.11114

[13] Lack, J., Gillard, M., Cam, M., et al. (2017) Circulating Tumor Cells Capture Disease Evolution in Advanced Prostate Cancer. Journal of Translational Medicine, 15, Article No. 44. https://doi.org/10.1186/s12967-017-1138-3

[14] Nel, I., Gauler, T.C., Bublitz, K., et al. (2016) Circulating Tumor Cell Composition in Renal Cell Carcinoma. PLoS ONE, 11, e153018. https://doi.org/10.1371/journal.pone.0153018

[15] Cristofanilli, M., Pierga, J.Y., Reuben, J., et al. (2019) The Clinical Use of Circulating Tumor Cells (CTCs) Enumeration for Staging of Metastatic Breast Cancer (MBC): International Expert Consensus Paper. Critical Reviews in Oncology/Hematology, 134, 39-45. https://doi.org/10.1016/j.critrevonc.2018.12.004

[16] Castello, A., Carbone, F.G., Rossi, S., et al. (2020) Circulating Tumor Cells and Metabolic Parameters in NSCLC Patients Treated with Checkpoint Inhibitors. Cancers (BaseI), 12, 487. 